Pacific Journal of Mathematic 


\title{
FACTORIZATION-PRIME IDEALS IN INTEGRAL DOMAINS
}

\author{
Helen E. Adams
}

\begin{abstract}
In this paper we characterize a new set of prime ideals of an integral domain $D$, called the set of $F$-primes of $D$, that we show to have the following properties: (1) $D$ is the intersection of all $D_{P}$ where $P$ is an $F$-prime of $D ;(2)$ all principal prime ideals and all essential prime ideals (those prime ideals for which $D_{P}$ is a valuation domain) are $F$-primes; (3) if $D$ is a GCD-domain, then the $F$-primes of $D$ are precisely the essential primes of $D$; (4) $D$ is a UFD if and only if the set of $F$-primes is precisely the set of principal prime ideals of $D$.
\end{abstract}

Introduction. In this paper we show that in many considerations involving both divisibility and prime ideals of an integral domain $D$, one can restrict attention to those prime ideals which contain no "relatively prime" elements of $D$ - that is, nonzero elements $a, b$ of $D$ whose only common divisors are units and whose only common multiples are multiples of $a b$. Kaplansky's "maximal primes of principal ideals" [2, p. 34] have a closely related property, since each such prime ideal $P$ contains no elements which are relatively prime to a fixed element of $P$. And Sheldon [3] has shown that, if $D$ is a GCD-domain, then the essential prime ideals are precisely those prime ideals which contain no relatively prime elements.

Section 1 contains the definition of this class of primes, which we call "factorization" primes, or " $F$-primes" for short, as well as a characterization of the essential prime ideals of $D$ (that is, those prime ideals $P$ of $D$ for which $D_{P}$ is a valuation domain) in a way that shows they are $F$-primes. The section ends with an alternate characterization of $F$ primes which shows that in a GCD-domain, the $F$-primes are just the essential primes.

Section 2 looks at the structure of a domain $D$ in terms of the set of $F$-primes of $D$. We define an " $F$-ideal" of $D$ (in such a way that the prime $F$-ideals are precisely the $F$-primes defined earlier) and show that every principal prime ideal of $D$ is an $F$-prime (although principal primes are not necessarily essential). The central result of this section shows that one of the basic properties of the set of all prime ideals of $D$ is satisfied by the set of $F$-primes of $D$; specifically the complement of a saturated multiplicatively closed subset of $D$ is a union of $F$-primes of $D$. This has the immediate consequences that every nonunit of $D$ is contained in an $F$-prime of $D$; that every minimal prime ideal of $D$ is an 
$F$-prime; and that $D$ is a UFD if and only if every $F$-prime of $D$ is principal. Finally we show that $\bigcap_{P \in \mathscr{F}} D_{P}=D$ for the set $\mathscr{F}$ of $F$-primes of $D$. Thus the $F$-primes of $D$ are just the essential primes only if $D$ is integrally closed.

While we have noted that every principal prime ideal of a Prüfer or GCD-domain is essential, in the concluding section we construct an example of a domain possessing a principal (and thus $F_{-}$-) prime ideal which is not essential. This domain is also interesting in that it contains two principal ideals whose intersection is not finitely generated.

1. Definitions and basic results. In this section and the next, $D$ is an integral domain. Let $a, b \in D$. Define $a$ and $b$ to be relatively prime in $D$, and write $(a, b)=1$, to mean $(a) \cap(b)=(a b)-$ that is, $a$ and $b$ have only trivial common multiples in $D$. Note that if $(a, b)=1$, then the only common divisors of $a$ and $b$ are units, but the converse is not true. For example, if $D$ is the ring of integers of $Q(\sqrt{-5})$ and $a=2, b=1+\sqrt{-5}$, then the only common divisors of $a$ and $b$ are units. But 6 is a nontrivial common multiple of 2 and $1+\sqrt{-5}$, and so $a$ and $b$ are not relatively prime according to our definition. If $D$ is a GCD-domain however, then $(a, b)=1$ if and only if $\operatorname{GCD}\{a, b\}=1$ (see Gilmer [1, p. 76]).

An $F$-prime of $D$ is a prime ideal of $D$ which contains no relatively prime elements of $D$. Thus the prime ideal $P$ of $D$ is an $F$-prime if and only if $P$ satisfies the following condition: given any $a, b \in P-\{0\}$, there exists $c \in D-(b)$ such that $a c \in(b)$.

The following result shows that essential prime ideals of $D$ are always $F$-primes of $D$.

Proposition 1.1. Let $P$ be a prime ideal of $D$. Then $P$ is essential if and only if $P$ satisfies the following condition:

(1.1.1) given any $a, b \in P$, there exists $c \in D-P$ such that $a c \in(b)$ or $b c \in(a)$.

Proof. Let $P$ be an essential prime ideal of $D$. Let $a, b \in P$. If $a / b \in P$, then $a / b=d / c$ where $c, d \in D$ and $c \notin P$. Hence $a c \in$ (b). Otherwise $b / a \in D_{P}$ and there exists $c \in D-P$ such that $b c \in(a)$.

Conversely, let $P$ satisfy (1.1.1). Let $a, b \in D$. If $a \notin P$ or $b \notin P$, then $b / a$ or $a / b \in D_{P}$, and we are done. So let $a, b \in P$. If there exists $c \in D-P$ such that $a c \in(b)$, then $a / b \in D_{P}$. Otherwise $b / a \in D_{P}$. Thus $D_{P}$ is a valuation domain and so $P$ is an essential prime.

Corollary 1.2. Every essential prime ideal of a domain $D$ is an F-prime of $D$. 
COROllary 1.3. In a Prüfer domain, the essential prime ideals and the F-prime ideals coincide.

Proof. The corollary follows since every prime ideal of a Prüfer domain is essential.

We shall see later that every $F$-prime of a domain $D$ is essential only if $D$ is integrally closed.

The next result provides a useful characterization of $F$-primes and relates $F$-primes to Sheldon's $P F$-primes of GCD-domains.

Proposition 1.4. Let $P$ be a prime ideal of an integral domain $D$. Then $P$ is an F-prime of $D$ if and only if $P$ satisfies the following property:

(1.4.1) if $t, x, y \in D$ such that $t x, t y \in P$ and $(x, y)=1$, then $t \in P$.

Proof. Let $P$ be an $F$-prime of $D$. Let $t, x, y \in D$ such that $t x, t y \in P$. If $t \notin P$, then $x, y \in P$ which contradicts $(x, y)=1$.

Conversely, let $P$ satisfy (1.4.1). Let $a, b \in P-\{0\} . \quad$ If $(a, b)=1$, then $1 \in P$. Thus $P$ is an $F$-prime of $D$.

Corollary 1.5. Let $D$ be a GCD-domain. Then the F-primes of $D$ are just the essential primes of $D$ (Sheldon's $P F$-primes [3]).

Proof. Let $t, x, y \in D$ such that $(x, y)=1$. Then $\operatorname{GCD}\{t x, t y\}=t$ (Kaplansky [2, p. 32]). Hence Proposition 1.4 shows that the $F$-primes of $D$ are precisely Sheldon's $P F$-primes of $D$ [3, Definition 2.1, p. 99] which in turn are just the essential prime ideals of $D[3$, Theorem 2.2, p. 99].

A more direct proof would be that every essential prime ideal is an $F$-prime of $D$ by Corollary 1.2 , and it is a straightforward exercise to show that every $F$-prime of $D$ is essential in this case.

2. Structure of a domain using F-primes. To construct $F$-primes for any integral domain $D$ and to show that $\bigcap_{P \in \mathscr{F}} D_{P}=D$, we need the concept of an " $F$-ideal". Define an $F$-ideal of $D$ to be an ideal $J$ of $D$ with the following property: if $a, b, d \in D$ such that $a d, b d \in J$ and $(a, b)=1$, then $d \in J$. From Proposition 1.4, F-primes of $D$ are the same as prime $F$-ideals of $D$.

Proposition 2.1. Every principal ideal of $D$ is an F-ideal. 
Proof. Let $c \in D$. Let $a, b, d \in D$ such that $a d, b d \in(c)$ and $(a, b)=1$. Then $a d=c \alpha$ and $b d=c \beta$ where $\alpha, \beta \in D$. Hence $a \beta=$ $b \alpha$ and so $\alpha \in(a)$ since $(a, b)=1$. Thus $d \in(c)$.

COROllary 2.2. Every principal prime ideal of $D$ is an F-prime.

Note that while every principal prime of a Prüfer or GCD-domain is essential (Corollaries 1.5 and 2.2), the example in $\$ 3$ is of a domain containing a principal prime ideal which is not essential.

Let $B$ be an ideal of $D$. We shall show that $B$ is contained in an $F$-ideal of $D$, and we shall construct the smallest such $F$-ideal. Note that while $D$ is not an $F$-prime ideal, $D$ is an $F$-ideal.

Let $A \subseteq D$. Define

$A^{\prime}=\{x \in D:$ there exists $a, b \in D$ such that $a x, b x \in A$

$$
\text { and }(a, b)=1\}
$$

Then $A \subseteq A^{\prime}$ since $(1,1)=1$. Denote the set of nonnegative integers by $N$. Define $B^{0}=B$ and, for each $n \in N, B^{n+1}=\left(B^{n}\right)^{\prime}$. Define $\bar{B}=\bigcup_{n \in N} B^{n}$. We shall use the following lemmas to show that $\bar{B}$ is the promised smallest $F$-ideal of $D$ which contains $B$.

Lemma 2.3. Let $n \in N$. Then $B^{n}$ is closed under multiplication with elements in $D$.

Proof. (By induction) The lemma holds for $n=0$. Assume that the lemma holds for $n=k$. Let $x \in B^{k+1}, y \in D$. Then there exist $a, b \in D$ such that $(a, b)=1$ and $a x, b x \in B^{k}$. Now, by assumption, $a x y, b x y \in B^{k}$. Hence $x y \in B^{k+1}$ by the definition of $B^{k+1}$. The lemma follows by induction.

\section{Lemma 2.4. Let $x, y \in B^{n}$. Then $x-y \in B^{2 n}$.}

Proof. (By induction) The lemma is true for $n=0$. Assume the result for $n=k$. Let $x, y \in B^{k+1}$. Then there exist $a, b, c, d \in D$ such that $a x, b x, c y, d y \in B^{k}$ and $(a, b)=1,(c, d)=1$. By Lemma 2.3, $a c x, a c y \in B^{k}$. Hence, by assumption, $a c(x-y) \in B^{2 k}$. Similarly $a d(x-y), \quad b c(x-y), \quad b d(x-y) \in B^{2 k}$. Hence $a(x-y), b(x-y) \in$ $B^{2 k+1}$ since $(c, d)=1$. Thus $(x-y) \in B^{2(k+1)}$ since $(a, b)=1$. The lemma follows by induction. 
Proposition 2.5. $\bar{B}$ is an F-ideal of $D$ which contains $B$. If $B \subseteq J$ where $J$ is an $F$-ideal of $D$, then $\bar{B} \subseteq J$.

Proof. The two preceding lemmas show that $\bar{B}$ is an ideal, and the construction of $\bar{B}$ guarantees that it is an $F$-ideal containing $B$. Furthermore, if $J$ is an $F$-ideal of $D$ and $A$ is an ideal of $D$ contained in $J$, then by definition, $A^{\prime} \subseteq J$. Hence if $B \subseteq J$, then $B^{n} \subseteq J$ for all $n$; and therefore $\bar{B} \subseteq J$.

The following lemma helps us prove the basic structural results for $F$-primes of $D$.

Lemma 2.6. Let $A$ be an F-ideal and $B$ be an ideal of $D$. If there exist $s \in \bar{B}, t \in D$ such that st $\notin A$, then there exists $b \in B$ such that $b t \notin A$.

Proof. (By induction) There exists $m \in N$ such that $s=$ $b_{m} \in B^{m}$. Now there exist $e, f \in D$ such that $(e, f)=1$ and $e b_{m}, f b_{m} \in B^{m-1}$. If $e b_{m} t, f b_{m} t \in A$, then $b_{m} t \in A$ since $A$ is an $F$ ideal. Hence $e b_{m} t \notin A$ or $f b_{m} t \notin A$ : that is, there exists $b_{m-1} \in B^{m-1}$ such that $b_{m-1} t \notin A$. Thus by induction there exists $b \in B^{0}=B$ such that $b t \notin A$.

THEOREM 2.7. Let $S$ be a multiplicatively closed subset of $D$. Let $A$ be maximal in the set of F-ideals of $D$ disjoint from $S$. Then $A$ is an $F$-prime of $D$.

Proof. Let $B$ and $C$ be ideals of $D$ which properly contain $A$. Then $\bar{B}$ and $\bar{C}$ meet $S$. Let $s \in \bar{B} \cap S$ and $t \in \bar{C} \cap S$. Then st $\notin A$. Hence by Lemma 2.6, there exists $b \in B$ such that $b t \notin A$ and, again by Lemma 2.6, there exists $c \in C$ such that $b c \notin A$. Thus $A$ is a prime ideal of $D$, which completes the proof.

COROllary 2.8. Every proper F-ideal of $D$ is contained in an F-prime ideal of $D$.

Proof. It is easily checked that the union of a chain of proper $F$-ideals of $D$ is a proper $F$-ideal of $D$. The corollary follows by Zorn's lemma.

THEOREM 2.9. Let $S$ be a saturated multiplicatively closed subset of $D$. Then the complement of $S$ is a union of F-primes of $D$.

Proof. Let $x \in D-S$. Then $(x) \subseteq D-S$ since $S$ is 
saturated. Since $(x)$ is an $F$-ideal of $D^{-}$(Proposition 2.1), then by Zorn's lemma, $(x)$ is contained in an $F$-ideal $J$ which is maximal in the set of $F$-ideals of $D$ disjoint from $S$. By Theorem 2.7, $J$ is an $F$-prime of $D$, which proves the theorem. of $D$.

Corollary 2.10. Every minimal prime ideal of $D$ is an F-prime

THEOREM 2.11. D is a UFD if and only if every F-prime of $D$ is principal.

Proof. Let $D$ be a UFD. Then by Corollary 1.5, every $F$-prime is essential and hence, by the properties of a UFD, is principal.

Conversely, if every $F$-prime of $D$ is principal, then by Theorem 2.9, every non-zero prime ideal of $D$ contains a principal prime. Thus $D$ is a UFD [2, Theorem 5, p. 4].

The following portion of an exercise from Kaplansky [2, Exercise 20, p. 42] gives insight as to why $\bigcap_{P \in \mathscr{F}} D_{P}=D$, where $\mathscr{F}$ is the set of $F$-primes of $D$. Recall that if $A$ and $B$ are ideals of $D$, then the set $B: A$ is defined to be $\{x \in D: x A \subseteq B\}$, which is an ideal of $D$.

PROPOSITION 2.12. Let $\mathscr{P}$ be a set of prime ideals of $D$ which satisfies the following property: if $a, b \in D$ such that $a \notin(b)$, then there exists $P \in \mathscr{P}$ such that $(b):(a) \subseteq P$. Then $\bigcap_{P \in \mathscr{P}} D_{P}=D$.

Proof. Now $D \subseteq \bigcap_{P \in \mathscr{P}} D_{P}$ since, for each $P \in \mathscr{P}, D \subseteq D_{P}$. Let $a, b \in D$ such that $a / b \in \bigcap_{P \in \mathscr{P}} D_{P} . \quad$ Let $P \in \mathscr{P}$. Then there exist $c, d \in$ $D$ such that $a / b=c / d$ and $d \notin P$. Now $a d \in(b)$, and so $(b):(a) \not \subset P$. Thus $a \in(b)$, and so $a / b \in D$. Hence $D=\bigcap_{P \in \mathscr{P}} D_{P}$.

Lemma 2.13. Let $a, b \in D$ such that $a \notin(b)$. Then $(b):(a)$ is $a$ proper $F$-ideal of $D$.

Proof. Clearly $(b):(a)$ is a proper ideal of $D$. Let $t x, t y \in(b):(a)$ where $t, x, y \in D$ and $(x, y)=1$. Then there exist $\alpha, \beta \in D$ such that $t x a=b \alpha$ and $t y a=b \beta$. Hence $t x a \beta=t y a \alpha$ : that is $x \beta=y \alpha$. Hence $\alpha \in(x)$ since $(x, y)=1$, and so $t a \in(b)$. Thus $t \in(b)$ : $(a)$, which completes the proof.

Lemma 2.13, Corollary 2.8 and Proposition 2.12 give us:

THEOREM 2.14. Let $\mathscr{F}$ be the set of F-prime ideals of a domain D. Then $D=\bigcap_{P \in \mathscr{F}} D_{P}$. 
Corollary 2.15. $D$ is integrally closed if and only if $D_{P}$ is integrally closed for each $F$-prime $P$ of $D$.

Proof. Kaplansky [2, Theorems 51 and 52, pp. 33 and 34].

COROllary 2.16. Let $D$ be an integral domain for which every $F$-prime is essential. Then $D$ is integrally closed.

Proof. For each $F$-prime $P$ of $D, D_{P}$ is a valuation ring which is integrally closed.

3. Example. Thanks are due to Phyllis Cassidy for suggesting examples of the following type occurring in differential algebra.

Let $R=Q[x, y, z]$ where $x, y, z$ are indeterminates. For each $n \in N$, define $D_{n}=Q\left[z, x / z^{n}, y / z^{n}\right]$, an integral domain lying between $R$ and its quotient field $Q(x, y, z)$. Define $D=\cup_{n \in N} D_{n}$ which is a subdomain of $Q(x, y, z)$ since, for each $n \in N, D_{n} \subseteq D_{n+1}$.

Lemma 3.1. Let $a \in D$. Then

(i) there exists $m \in N$ such that $z^{m} a \in R$;

(ii) there exists $q \in Q$ such that $a-q \in(z)$;

(iii) $1 / z \notin D$.

Proof. Since $a \in D$, there exists $n \in N$ such that $a \in D_{n}$. Hence

$$
a=\sum_{i=0}^{k} q_{t} z^{\alpha_{i}}\left(x / z^{n}\right)^{\beta_{i}}\left(y / z^{n}\right)^{\gamma_{i}}
$$

where $q_{i} \in Q ; k, \alpha_{\imath}, \beta_{\imath}, \gamma_{\imath} \in N$; and like terms have been collected.

(i) Let $m=\max _{0 \leqq ı k}\left\{n\left(\beta_{i}+\gamma_{1}\right)-\alpha_{i}\right\}$. Then $z^{m} a \in R$.

(ii) Since, for each $h \in N, x / z^{h}=\left(x / z^{h+1}\right) z$ and $y / z^{h}=\left(y / z^{h+1}\right) z$, then $x / z^{h}, y / z^{h} \in(z)$. If, for each $i \in\{0, \cdots, k\}, \alpha_{\imath} \geqq 1$ or $\beta_{\imath} \geqq 1$ or $\gamma_{i} \geqq 1$, then $a \in(z)$. Otherwise there exists $j \in\{0, \cdots, k\}$ such that $\alpha_{\jmath}=\beta_{j}=\gamma_{\jmath}=0$ and so $a=q_{j}+b$ where $b \in(z)$.

(iii) Suppose $a=1 / z$. Then using the number $m$ from part (i), we have that

$$
z^{m-1}=z^{m} a=\sum_{i=0}^{k} q_{i} z^{m+\alpha_{i}-n\left(\beta_{i}+\gamma_{i}\right)} x^{\beta_{i}} y^{\gamma_{i}}
$$

which is an equation in $R$. Since the right-hand expression equals the monomial $z^{m-1}$, it must have a single nonzero term, say the 0 th term, with both $\beta_{0}$ and $\gamma_{0}=0$. But then $m+\alpha_{0}=m-1$ and hence $\alpha_{0}=-1$, which contradicts the requirement that $\alpha_{0}$ be a positive integer. Hence $1 / z \notin D$. 
Proposition 3.2. The ideal $(z)$ is an F-prime of $D$, yet $D_{(z)}$ is not a valuation domain.

Proof. (i) Now $(z) \neq D$ since $1 / z \notin D$ by Lemma 3.1(iii). Let $I$ be an ideal of $D$ properly containing ( $z$ ). Then by Lemma 3.1(ii), there exists $a \in I$ such that $a=q+b$ where $q \in Q, q \neq 0, b \in(z)$. Since $q^{-1} \in D, a q^{-1}=1+b q^{-1} \in I$. Thus $1 \in I$ since $b q^{-1} \in(z) \subseteq I$. Hence $(z)$ is a maximal ideal of $D$, and thus $(z)$ is an $F$-prime of $D$ by Corollary 2.2 .

(ii) We shall show that $x / y \notin D_{(z)}$ and $y / x \notin D_{(z)}$. Let $x / y=a / b$ where $a, b \in D$. Then $a y=b x$. By Lemma 3.1(i), there exist $m, n \in N$ such that $z^{m} a, z^{n} b \in R$. Now $z^{n}\left(z^{m} a\right) y=z^{m}\left(z^{n} b\right) x$ which is an equation in $R$. Hence $y$ divides $z^{n} b$ and $x$ divides $z^{m} a$ in $R$, and so $y / z^{n}$ divides $b$ and $x / z^{m}$ divides $a$ in $D$. Thus $a, b \in(z)$ since $y / z^{n}, x / z^{m} \in$ $(z)$. This completes the proof that $D_{(z)}$ is not a valuation domain.

Proposition 3.3. In the domain $D$, the ideal $(x) \cap(y)$ is not finitely generated.

Proof. Let $\alpha \in(x) \cap(y)$. Then by Lemma 3.1(i), there exist $a, b \in R$ and $h, m \in N$ such that $\alpha=x\left(a / z^{h}\right)=y\left(b / z^{m}\right)$. Rewriting this as an equation in $R$, we find that $x$ divides $b$ in $R$. Thus there exists $c \in R$ such that $\alpha=x y c / z^{m}$. Suppose that $(x) \cap(y)$ is the finitely generated ideal $\left(\alpha_{1}, \cdots, \alpha_{k}\right)$ where $\alpha_{i} \in D$. Then, for each $\alpha_{i}$, there exists $m_{\imath} \in N$ such that $\alpha_{\imath} \in\left(x y / z^{m_{i}}\right)$. Let $M=\max _{1 \leqq i \leqq k}\left\{m_{i}\right\}$. Then each $\alpha_{i} \in\left(x y / z^{M}\right)$. Hence $\left(\alpha_{1}, \cdots, \alpha_{k}\right) \subseteq\left(x y / z^{M}\right)$. Now $x y / z^{M+1} \notin\left(x y / z^{M}\right)$ since $1 / z \notin D$. But $x y / z^{M+1} \in(x) \cap(y)$ which contradicts the supposition. Hence $(x) \cap(y)$ is not finitely generated.

\section{REFERENCES}

1. R. Gilmer, Multiplicative Ideal Theory, Marcel Dekker, New York, 1972.

2. I. Kaplansky, Commutative Rings, University of Chicago Press, Chicago, 1974.

3. P. Sheldon, Prime ideals in GCD domains, Canad. J. Math., 26 (1974), 98-107.

Received February 23, 1976 and in revised form June 2, 1976.

SMith College 



\section{Pacific Journal of Mathematics}

\section{Vol. 66, No. $1 \quad$ November, 1976}

Helen Elizabeth. Adams, Factorization-prime ideals in integral domains ............ Patrick Robert Ahern and Robert Bruce Schneider, The boundary behavior of Henkin's kernel.

Daniel D. Anderson, Jacob R. Matijevic and Warren Douglas Nichols, The Krull

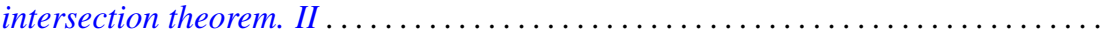

Efraim Pacillas Armendariz, On semiprime P.I.-algebras over commutative regular

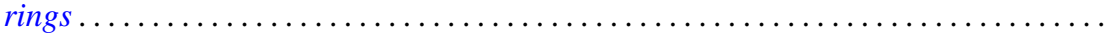

Robert H. Bird and Charles John Parry, Integral bases for bicyclic biquadratic fields

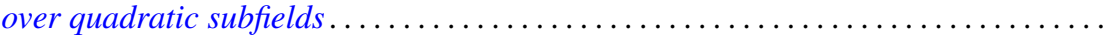

Tae Ho Choe and Young Hee Hong, Extensions of completely regular ordered

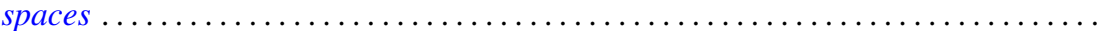

John Dauns, Generalized monoform and quasi injective modules ...............

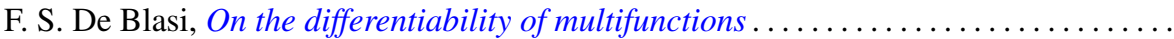

Paul M. Eakin, Jr. and Avinash Madhav Sathaye, R-endomorphisms of $R[[X]]$ are

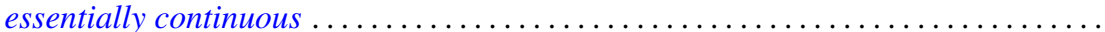

Larry Quin Eifler, Open mapping theorems for probability measures on metric

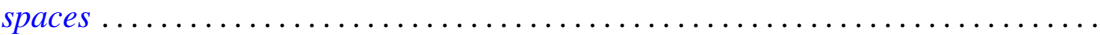

Garret J. Etgen and James Pawlowski, Oscillation criteria for second order self adjoint

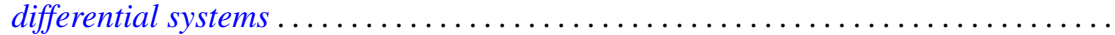

Ronald Fintushel, Local $S^{1}$ actions on 3-manifolds .

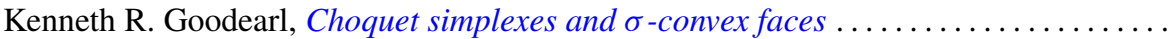

John R. Graef, Some nonoscillation criteria for higher order nonlinear differential

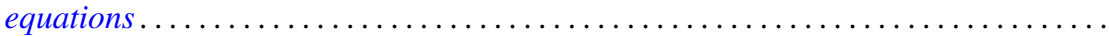

Charles Henry Heiberg, Norms of powers of absolutely convergent Fourier series: an example.

Les Andrew Karlovitz, Existence of fixed points of nonexpansive mappings in a space

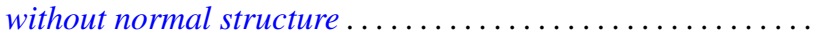

Gangaram S. Ladde, Systems of functional differential inequalities and functional

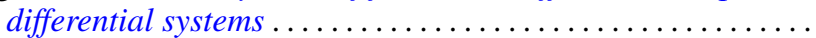

Joseph Michael Lambert, Conditions for simultaneous approximation and interpolation with norm preservation in $C[a, b]$.

Ernest Paul Lane, Insertion of a continuous function.

Robert F. Lax, Weierstrass points of products of Riemann surfaces .

Dan McCord, An estimate of the Nielsen number and an example concerning the Lefschetz fixed point theorem...

Paul Milnes and John Sydney Pym, Counterexample in the theory of continuous functions on topological groups...

Peter Johanna I. M. De Paepe, Homomorphism spaces of algebras of holomorphic functions

Judith Ann Palagallo, A representation of additive functionals on $L^{p}$-spaces,

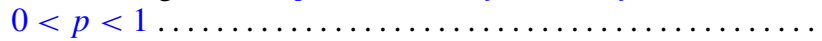

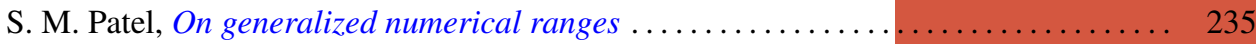

Thomas Thornton Read, A limit-point criterion for expressions with oscillatory

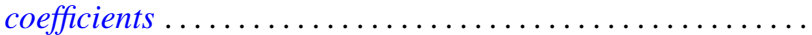

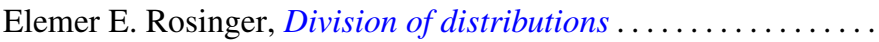

Peter S. Shoenfeld, Highly proximal and generalized almost finite

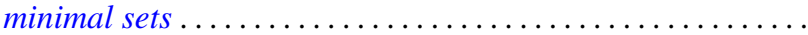

R. Sirois-Dumais and Stephen Willard, Quotient-universal sequential spaces

Robert Charles Thompson, Convex and concave functions of singular values of matrix sums....

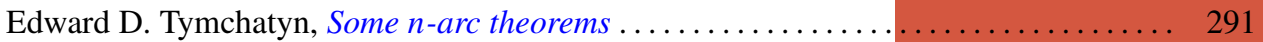

